\title{
An Optimization Strategy for Customized Radiotherapy Head Immobilization Masks
}

\author{
D.S. Craveiro, M.A.R. Loja, L. Vieira, M. Vinyas
}

\begin{abstract}
An effective head immobilization is an important requirement in radiotherapy treatment sessions, although it may also be thought in the future as a precious aid in brain medical imaging. Thus, the present work is focused on the stiffness optimization of a customized head immobilization mask, modelled upon the head reconstruction surface based on computerized tomography images.

This paper proposes a strategy supported by a metaheuristic optimization technique and a metamodeling approach for the whole mask, illustrated at its most unfavorable region occurring in the gnathion region.
\end{abstract}

\section{INTRODUCTION}

Customized devices may be an important contribution to the improvement of radiotherapy immobilization devices. The recent years' evolution of reverse engineering techniques may contribute to the development of new interdisciplinary research areas, namely involving medicine and engineering areas and finding useful solutions for specific problems as the present work intends to.

The improvement of the actual construction process of the immobilization masks as well as their configuration, can lead to better health care experience for the patient [1]. Also the type of technology available for the modelling of surfaces using CT imaging or other simpler acquisition techniques, that perform better and with less radiation risks for the subjects' health can be a quality gain, through the increase of the ratio benefits/risk for the patient [2].

From the literature review it is possible to conclude that the combination of acquisition and reconstruction techniques as well as the subsequent modelling can provide effectively advantageous purpose-oriented customized devices as presented in [3] - [6]. However, it may be concluded that an extensive work is still needed. Illustrating this, it is worth

D.S. Craveiro is with GIMOSM - Grupo de Investigação em Modelação e Optimização de Sistemas Multifuncionais, ISEL/IPL - Instituto Politécnico de Lisboa, Av. Conselheiro Emídio Navarro 1, 1959-007 Lisboa, Portugal (email: deboraalexandracraveiro@gmail.com).

M.A.R. Loja is with GIMOSM - Grupo de Investigação em Modelação e Optimização de Sistemas Multifuncionais, ISEL/IPL - Instituto Politécnico de Lisboa, and with IDMEC/IST - Instituto de Engenharia Mecânica, Av. Rovisco Pais, 1049-001 Lisboa, Portugal (email: amelialoja@tecnico.ulisboa.pt).

L. Vieira is with GIMOSM - Grupo de Investigação em Modelação e Optimização de Sistemas Multifuncionais, with H\&TRC - Health \&Technology Research Center, ESTeSL/IPL - Instituto Politécnico de Lisboa, Av. D. João II, Lote 4.69.01, 1990-096 Lisboa, Portugal and with IBEB-Instituto de Biofísica e Engenharia Biomédica, Faculdade de Ciências da Universidade de Lisboa, Lisboa, Portugal (e-mail: lina.vieira@estesl.ipl.pt).

M. Vynias is with Dept. Mechanical Engineering, Nitte Meenakshi Institute of Technology, Bangalore, India - 560064 (e-mail: vinyas.mahesh@gmail.com). mentioning that no works were found on the establishment of a procedure yielding optimal customized head immobilization masks supported by a mechanical analysis. Thus this study constitutes a first approach in this direction, presenting a possible optimization strategy in this context.

\section{MATERIALS AND METHODS}

This section presents a brief overview of the fundamentals considered in the present study.

\section{A. Geometrical Modelling}

The surface of the mask used in the present work was obtained by a reverse engineering process, which started with the images acquisition via a Computed Tomography (CT) to the head of physical phantom (PIXYTM) PIXY is an anatomic and radiologic anthropomorphic phantom to training/teaching mainly used in Radiological Procedures. The images obtained were converted into polygonal mesh files which were then imported into SolidWorks ${ }^{\circledR}$ using its ScanTo3D utility to reconstruct the surfaces and finally to design the mask[7]. The present customized designed mask is open in the eyes and mouth to provide more comfort and to minimize claustrophobic sensation in the patients. The fixture of the mask to the radiotherapy table is provided via a U-shaped scheme horizontally fixed to the mask surface (Figure 1).

Figure 1. Immobilization mask to optimize

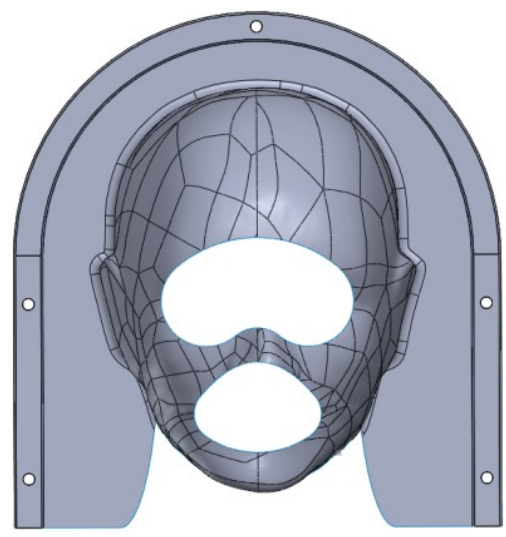

The strength, stiffness and material selection studies were already performed in [6],[7]. Presently this immobilization device will be optimized via a hybrid metaheuristic approach, in order to obtain an optimal configuration concerning to its stiffness.

\section{B. The Whale Optimization Algorithm}

The Whale Optimization Algorithm (WOA) is a metaheuristic optimization algorithm that mimics the humpback whales predatory behavior [8]. The original pseudo-code is adapted in Figure 2. 
Figure 2. WOA adapted pseudo-code[8]

Initialize whale population $\mathrm{X}_{\mathrm{i}}(\mathrm{i}=1,2, \ldots, \mathrm{n})$

Calculate the fitness of each search agent $X_{i}$

$\mathrm{X}^{*}=$ the best search agent

while ( $t<$ maximum number of iterations)

for each search agent

Update a, A, C, 1 and $\mathrm{p}$

if1 $(\mathrm{p}<0.5)$

if2 $(|\mathrm{A}|<1)$

$\overrightarrow{\mathrm{D}}=\left|\overrightarrow{\mathrm{C}} \cdot \overrightarrow{\mathrm{X}^{*}}(\mathrm{t})-\overrightarrow{\mathrm{X}}(\mathrm{t})\right|$

$\vec{X}(t+1)=\overrightarrow{X^{*}}(t)-\vec{A} \cdot \vec{D}$

else if $2(|A| \geq 1)$

Select a random search agent $\left(X_{\text {rand }}\right)$

$\overrightarrow{\mathrm{D}}=\left|\overrightarrow{\mathrm{C}} \cdot \overrightarrow{X_{\text {rand }}}(\mathrm{t})-\overrightarrow{\mathrm{X}}(\mathrm{t})\right|$

$\overrightarrow{\mathrm{X}}(\mathrm{t}+1)=\overrightarrow{X_{\text {rand }}}(\mathrm{t})-\overrightarrow{\mathrm{A}} \cdot \overrightarrow{\mathrm{D}}$

end if 2

else if1 $(p \geq 0.5)$

$$
\overrightarrow{\mathrm{D}^{\prime}}=\left|\overrightarrow{\mathrm{X}^{*}}(\mathrm{t})-\overrightarrow{\mathrm{X}}(\mathrm{t})\right|
$$

$\overrightarrow{\mathrm{X}}(\mathrm{t}+1)=\overrightarrow{\mathrm{D}^{\prime}} \cdot \mathrm{e}^{\mathrm{bl}} \cdot \cos (2 \pi \mathrm{l})+\overrightarrow{\mathrm{X}^{*}}(\mathrm{t})$

end if1

end for

Check if any search agent goes beyond the search

space and amend it

Calculate the fitness of each search agent

Update $X^{*}$ if there is a better solution

$\mathrm{t}=\mathrm{t}+1$

end while

return $X^{*}$

The $\vec{A}$ vector is calculated as $\vec{A}=2 \vec{a} \cdot \vec{r}-\vec{a}$ and the $\vec{C}$ vector is calculated as $\vec{C}=2 \cdot \vec{r}$, where $\vec{a}$ linearly decreases from 2 to 0 over the course of generations and $\vec{r}$ is a random vector in $[0,1]$. The $p$ value is a random number between 0 and 1 is also a random number in $[-1,1]$, and $b$ is constant that defines the spiral shape.

\section{Kriging Interpolation Method}

The Kriging interpolation method is commonly used in spatial interpolation problems. This method states that close data points will have similar behavior or properties [9].

To solve the interpolation problem, this method uses semivariograms. From the available functions to this purpose [9], [10], one has selected the one given in (1):

$$
\gamma(\mathrm{h})=\exp \left(-\frac{\mathrm{h}^{2}}{2 \mathrm{~L}^{2}}\right)
$$

where $h$ is the Euclidean distance. The estimated value in a certain point $j$ is then given by:

$$
\mathrm{z}_{\mathrm{j}}=\sum_{\mathrm{i}} \omega_{\mathrm{ji}} \mathrm{p}_{\mathrm{i}}
$$

being $p_{i}$ the known data points and $\omega_{\mathrm{ji}}$ the weights that result from the mentioned assumption that closer points will have a similar behavior. To calculate these weights, it is necessary to solve equation (3), that relates the covariance matrix $(\Gamma)$, the weights $\left(\Omega_{\mathrm{j}}\right)$ and the semivariogram $\left(\alpha_{\mathrm{j}}\right)$ between an unknown point and the known data points:

$$
\left[\begin{array}{ccc}
\gamma_{11} & \cdots & \gamma_{1 \mathrm{n}} \\
\vdots & \ddots & \vdots \\
\gamma_{\mathrm{n} 1} & \cdots & \gamma_{\mathrm{nn}}
\end{array}\right]\left[\begin{array}{c}
\omega_{\mathrm{j} 1} \\
\vdots \\
\omega_{\mathrm{jn}}
\end{array}\right]=\left[\begin{array}{c}
\gamma_{\mathrm{j} 1} \\
\vdots \\
\gamma_{\mathrm{jn}}
\end{array}\right] \Leftrightarrow \Gamma \cdot \Omega_{\mathrm{j}}=\alpha_{\mathrm{j}}
$$

In this study, one has considered two Kriging formulations, the Simple Kriging and the Common Kriging, which differ in solving the weights, as presented in [9],[10]:

$$
\left[\begin{array}{cccc}
\gamma_{11} & \cdots & \gamma_{1 \mathrm{n}} & 1 \\
\cdots & \ddots & \cdots & 1 \\
\gamma_{\mathrm{nn}} & \cdots & \gamma_{\mathrm{nn}} & 1 \\
1 & 1 & 1 & 0
\end{array}\right]\left[\begin{array}{c}
\omega_{\mathrm{j} 1} \\
\vdots \\
\omega_{\mathrm{jn}} \\
\lambda
\end{array}\right]=\left[\begin{array}{c}
\gamma_{\mathrm{j} 1} \\
\vdots \\
\gamma_{\mathrm{jn}} \\
1
\end{array}\right]
$$

\section{Optimization scheme validation}

To verify the effectiveness of the optimization approach which was thought to minimize the computational cost, and previous to its use on the immobilization masks, one has considered its application on a thin simply supported square plate submitted to a uniform load. The plate material was the same as the one used for the mask, the Polyphenylene Sulfide, previously considered in [7]. Considering to other parameters, the edges dimension was set to $100 \mathrm{~mm}$ and the applied load was $0.02 \mathrm{MPa}$. The yield point was ensured in the thickness optimization interval.

The objective of this preliminary optimization study was the minimization of the maximum displacement verified in a plate, when submitted to a load scenario, as a function of thickness. A similar objective to the one meant for the mask. The plate displacements were obtained via finite element method based on a high order shear deformation theory [11].

To minimize the plate maximum displacement as a function of the thickness, it was necessary to collect data from the nodes of the plate, which included the nodal coordinates $(\mathrm{x}, \mathrm{y})$, the corresponding nodal transverse displacements and the thickness at the point.

To obtain a better conforming behavior to the edges, one has considered every node located on the edges, $50 \%$ of the nodes from the interior of the plate and the node of maximum displacement. The thicknesses considered pertained to the set $t=\{2,2.5,3,3.5,4\}$. The optimization process was divided into two steps, the achievement of the maximum displacement and its location, and then the minimization of the maximum displacement.

\section{1) Maximum displacement location}

To obtain the location of the maximum displacement in the plate, for each thickness considered, the displacement surface was interpolated using Simple Kriging and a DUAL problem formulation of the surface, since the WOA is presented as a minimization algorithm. The number of generations used, were 150 with 30 whales each. The maximum displacement and its coordinates $(\mathrm{x}, \mathrm{y})$ location was found for each thickness. The expected location was exactly in the center of the plate however, the nodes used in the interpolation are not symmetrical which induced into slightly different values. In order to guarantee a fixed location, it was calculated an average between the obtained values of each thickness and a standard deviation.

\section{2) Minimization of the maximum displacement}

Considering the previous characterization of the maximum displacement for each plate thickness, one has then obtained a function of the minimum maximum 
displacement of the plate via the least squares method. This function was optimized using the WOA with 50 generations with a population of 30 whales each.

\section{E. Model to Optimize}

In the optimization of the immobilization mask, it was considered the left lateral bending of the neck as load case, which represents the worst case scenario. As the gnathion region was the most unfavorable, one has restricted the optimization study to this region, which is represented in red close to the "max" caption in Figure 3.

Figure 3. Considered zone for the optimization study of the mask

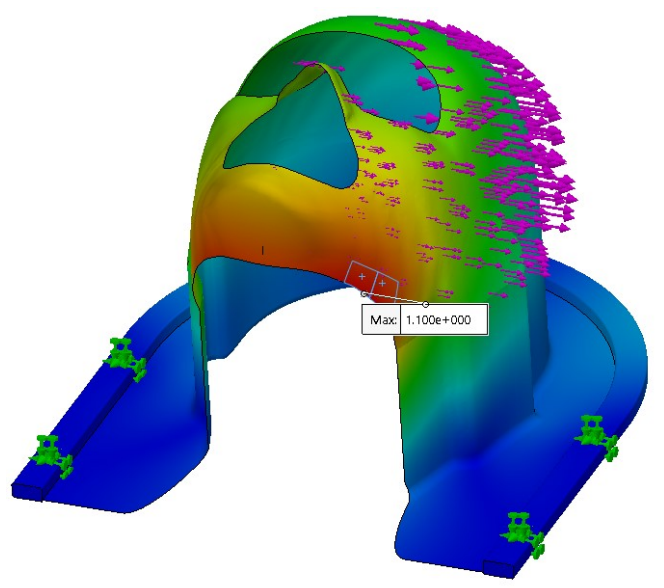

The data points considered are the nodes of the region of the Figure 3, the information includes the coordinates $(\mathrm{x}, \mathrm{y}, \mathrm{z})$ of each node, thickness and its corresponding displacement. The thicknesses considered were $\mathrm{t}=\{5,5.125,5.25,5.375$, $5.5\}$. As for the optimization of the thin plate, the method used to optimize the maximum displacement in the mask considered two steps. The first, the maximization of the displacement to find its location, interpolating the deformation as function of the spatial coordinates $(x, y, z)$ for each thickness using this time the Common Kriging, as from the testes performed one has concluded that this approach will provide better results. This function was optimized using the WOA with the same parameters as the ones used for the thin plate. In the second optimization step, the minimization of the maximum displacement, one has used the least squares to obtain the maximum displacements function, which was then minimized considering the WOA which, once again, used the same parameters as for the thin plate optimization study.

\section{RESULTS}

This section presents the results obtained, first for a single plate with the aim of constituting a methodology verification case, then the second study focuses the immobilization mask. In this first optimization work one has only considered lateral constraints.

\section{A. Verification case: Thin plate optimization}

For the minimization of the plate displacement, the expected values (Wmax) and the presently predicted values $\left(\mathrm{W}\left(\mathrm{X}^{*}, \mathrm{Y}^{*}\right)\right)$ using Simple Kriging interpolation method and WOA are presented in Table 1. The thickness is referred as $t$.
Table 1. Results of the displacement maximization of the plate

\begin{tabular}{|c|c|c|c|}
\hline $\mathbf{t}[\mathbf{m m}]$ & $\mathbf{W m a x}[\mathbf{m m}]$ & $\mathbf{W}\left(\mathbf{X}^{*}, \mathbf{Y}^{*}\right)[\mathbf{m m}]$ & Error $(\%)$ \\
\hline 2 & 2,368 & 2,352 & $-0,67683$ \\
\hline 2,5 & 1,214 & 1,206 & $-0,6802$ \\
\hline 3 & 0,704 & 0,699 & $-0,65611$ \\
\hline 3,5 & 0,444 & 0,441 & $-0,6425$ \\
\hline 4 & 0,298 & 0,296 & $-0,62992$ \\
\hline
\end{tabular}

The error is very small, even when not using symmetric data points. In Table 2 it is now possible to observe the error between the $\mathrm{W}\left(\mathrm{X}^{*}, \mathrm{Y}^{*}\right)$ and the maximum displacement obtained with the function obtained via the least squares method $\mathrm{W}_{\mathrm{X}^{*}, \mathrm{Y}^{*}}(\mathrm{t})$, which is again negligible.

Table 2. Results of the least squares method

\begin{tabular}{|c|c|c|c|}
\hline $\mathbf{t}[\mathbf{m m}]$ & $\mathbf{W}\left(\mathbf{X}^{*}, \mathbf{Y}^{*}\right)[\mathbf{m m}]$ & $\mathbf{W}_{\mathbf{X}^{*}, \mathbf{Y}^{*}}(\mathbf{t})[\mathbf{m m}]$ & Error $(\mathbf{\%})$ \\
\hline 2 & 2,352 & 2,352 & $-5,95 \mathrm{E}-05$ \\
\hline 2,5 & 1,206 & 1,206 & $4,72 \mathrm{E}-04$ \\
\hline 3 & 0,699 & 0,699 & $-1,21 \mathrm{E}-03$ \\
\hline 3,5 & 0,441 & 0,441 & $1,30 \mathrm{E}-03$ \\
\hline 4 & 0,296 & 0,296 & $-4,45 \mathrm{E}-04$ \\
\hline
\end{tabular}

This function $\mathrm{W}_{\mathrm{X}^{*}, \mathrm{Y}^{*}}(\mathrm{t})$ was optimized using the WOA presenting then the results in Table 3 and Figure 4:

Table 3. Results of the minimization of the maximum displacement

\begin{tabular}{|c|c|}
\hline Iteration $=\mathbf{5 0}$ & Optimization Results \\
\hline $\mathbf{W}_{\mathbf{X}^{*}, \mathbf{Y}^{*}}\left(\mathbf{t}^{*}\right)$ & 0,2963 \\
\hline $\mathbf{t}^{*}$ & 4 \\
\hline
\end{tabular}

Figure 4. Best solution and standard deviation evolution

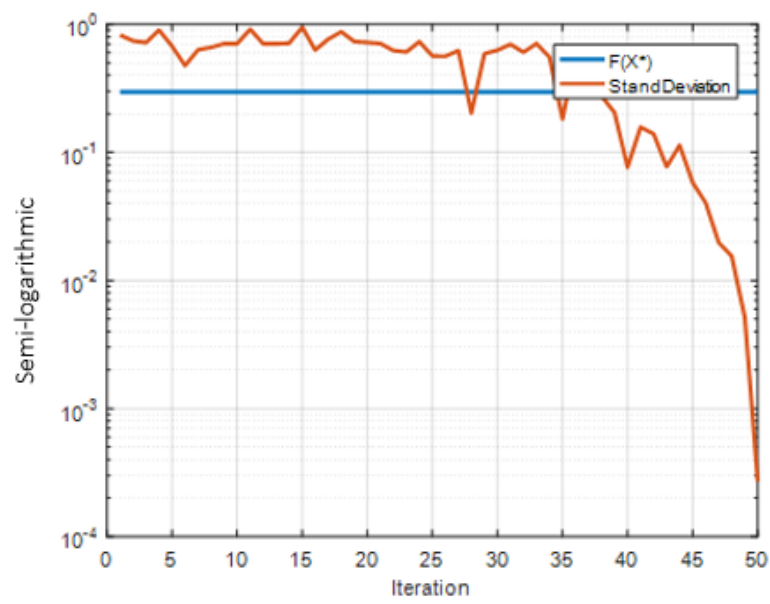

As it was expected the thickness was maximized (Table 3) and the WOA behavior over the course of the iterations is presented in Figure 4.

\section{B. Mask optimization}

Following a similar procedure, the maximum displacements of the masks are presented in the Table 4. 
Table 4. Displacement maximization of the mask

\begin{tabular}{|c|c|c|c|}
\hline $\mathbf{t}[\mathbf{m m}]$ & $\mathbf{W m a x}[\mathbf{m m}]$ & $\mathbf{W}\left(\mathbf{X}^{*}, \mathbf{Y}^{*}, \mathbf{Z}^{*}\right)[\mathbf{m m}]$ & Error $(\mathbf{\%})$ \\
\hline 5 & 1,1 & 1,1178 & 1,618182 \\
\hline 5,125 & 1,033 & 1,0507 & 1,713456 \\
\hline 5,25 & 0,9719 & 0,9898 & 1,841753 \\
\hline 5,375 & 0,9156 & 0,9335 & 1,955002 \\
\hline 5,5 & 0,8637 & 0,8818 & 2,095635 \\
\hline
\end{tabular}

Using these results to interpolate $\mathrm{W}_{\mathrm{X}^{*}, \mathrm{Y}^{*}, Z^{*}}(\mathrm{t})$ that represent the maximum displacement as function of the thickness, the results using the least squares method are presented in Table 5 .

Table 5. Results of the least squares method for the mask

\begin{tabular}{|c|c|c|c|}
\hline $\mathbf{t}[\mathbf{m m}]$ & $\mathbf{W}\left(\mathbf{X}^{*}, \mathbf{Y}^{*}, \mathbf{Z}^{*}\right)[\mathbf{m m}]$ & $\mathbf{W}_{\mathbf{X}^{*}, \mathbf{Y}^{*}, \mathbf{Z}^{*}}(\mathbf{t})[\mathbf{m m}]$ & Error $(\mathbf{\%})$ \\
\hline 5 & 1,1178 & 1,1177 & $-0,011$ \\
\hline 5,125 & 1,0507 & 1,0510 & 0,028 \\
\hline 5,25 & 0,9898 & 0,9897 & $-0,014$ \\
\hline 5,375 & 0,9335 & 0,9334 & $-0,012$ \\
\hline 5,5 & 0,8818 & 0,8819 & 0,008 \\
\hline
\end{tabular}

And finally, for the minimization of the maximum displacement of the mask, the results obtained are presented in Table 6, showing the minimum displacement and the corresponding thickness, and in Figure 5 the evolution of the WOA algorithm over the course of the iterations.

Figure 5. Best solution and standard deviation evolution

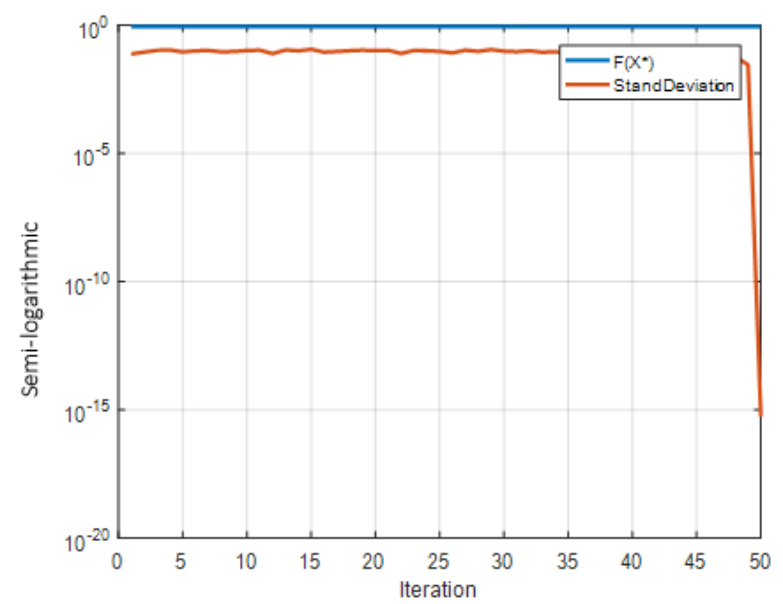

Table 6. Minimization of the mask maximum displacement

\begin{tabular}{|c|c|}
\hline Iteration $=\mathbf{5 0}$ & Optimization Results \\
\hline $\mathbf{W}_{\mathbf{X}^{*}, \mathbf{Y}^{*}, \mathbf{Z}^{*}}\left(\mathbf{t}^{*}\right)$ & 0,8819 \\
\hline $\mathbf{t}^{*}$ & 5,5 \\
\hline
\end{tabular}

As it was expected, the thickness increased with the minimization of the displacement. It is also visible a good performance concerning to the magnitude of the errors.

\section{CONCLUSION}

This study aimed at optimizing customized head immobilization masks, testing a hybrid approach based on a surrogate model and a metaheuristic optimization technique. From the results obtained, it can be concluded that the WOA possesses good characteristics concerning to the exploitation of the design variables space yielding good results. The kriging metamodeling technique, provides an effective tool for surfaces adjustment, however this is still a topic that needs further research in order to better characterize specific requirements for more complex surfaces as is the mask case. Globally it is possible to conclude that the optimization strategy developed is a promising approach to this subject.

\section{ACKNOWLEDGMENT}

The authors wish to acknowledge the financial support of Project IPL/2016/SoftImob/ISEL, and the support of FCT through IDMEC, LAETA, project UID/EMS/50022/2019.

The authors also wish to acknowledge Fundação Champalimaud the possibility to obtain the CT images which were essential to this study.

\section{REFERENCES}

[1] Li G, Lovelock DM, Mechalakos J, Rao S, Della-Biancia C, Amols H, Lee N. 2013. Migration from Full-Head Mask to 'open-Face' Mask for Immobilization of Patients with Head and Neck Cancer. Journal of Applied Clinical Medical Physics. 14(5): 243-54.

[2] Kerr W, Rowe P, Pierce SG. 2017. Accurate 3D Reconstruction of Bony Surfaces Using Ultrasonic Synthetic Aperture Techniques for Robotic Knee Arthroplasty. Computerized Medical Imaging and Graphics. 58: 23-32.

[3] J.M.Machado, M.S.Monteiro, V.F.Vieira, J.A.Collinot, J.O.Prior, L.Vieira, J.A.Pires-Jorge, "Value of a Lower-Limb Immobilization Device for Optimization of SPECT/CT Image Fusion," J. Nucl. Med. Technol., vol. 43, no. 2, pp. 98-102, 2015.

[4] M.Fisher, C.Applegate, M.Ryalat, S.Laycock, M.Hulse, D.Emmens, D.Bell, "Evaluation of 3-D Printed Immobilisation Shells for Head and Neck IMRT," December, pp.322-328, 2014.

[5] S. .Chen, Y.Lu, C.Hopfgartner, M.Sühling, S.Steidl, J.Hornegger, "3D printing based production of head and neck masks for radiation therapy using ct volume data : a fully automatic framework. (ISBI) 2016 IEEE 13th Int. Symposium Biomedical Imaging, vol. D, pp.403-406, 2016.

[6] M. Loja et al., "Using 3D Anthropometric Data for the Modelling of Costumized Head Immobilization Masks," Comput. Methods Biomech. Biomed. Eng. Imaging Vis., pp.1-42, 2018.

[7] D.S.Craveiro et al., "Immobilization devices for diagnosis and therapeutic medical applications," in XIII CIBEM - 2017 Lisboa, 2017, pp. 1-11.

[8] S.Mirjalili. A.Lewis, "the whale optimization algorithm", Adv. Eng. Softw., vol. 95, pp. 51-67, 2016.

[9] V. S. Fazio, M. Roisenberg, "Spatial interpolation: an analytical comparison netween kriging and RBF networks", Proc. 28th Annu. ACM Symp. Appl. Comput., vol.2(1), pp.2-7, 2013.

[10] M.A.R.Loja, J.I.Barbosa, C.M.Mota Soares, "Analysis of Sandwich Beam Structures Using Kriging Based Higher Order Models". Composite Structures, vol.119, pp.99-106, 2015.

[11] A.J.M. Ferreira, "Problemas de Elementos Finitos em MATLAB". Fundação Calouste Gulbenkian, 2010. 